\title{
PROPOSTA E IMPLEMENTAÇÃO EM PYTHON DO MÉTODO SIMPLE AGGREGATION OF PREFERENCES EXPRESSED BY ORDINAL VECTORS - MULTI DECISION MAKERS: UMA FERRAMENTA WEB SIMPLES E INTUITIVA PARA APOIO À DECISÃO MULTICRITÉRIO
}

\section{Luiz Frederico Horácio de Souza de Barros Teixeira}

Centro de Análises de Sistemas Navais (CASNAV)/ Universidade Federal Fluminense (UFF) Praça Barão de Ladário, s/nº Ilha das Cobras, Rua da Ponte, Ed. 23, Centro, Rio de Janeiro - RJ / Rua Passo da Pátria, 156 - 209, São Domingos, Niterói - RJ

frederico.horacio@gmail.com

\section{Marcos dos Santos}

Centro de Análises de Sistemas Navais (CASNAV) / Instituto Militar de Engenharia (IME) Praça Barão de Ladário, s/nº Ilha das Cobras, Rua da Ponte, Ed. 23, Centro, Rio de Janeiro - RJ / Praça Gen. Tibúrcio, nº 80, Praia Vermelha, Urca, Rio de Janeiro - RJ marcosdossantos doutorado@yahoo.com.br

Carlos Francisco Simões Gomes

Universidade Federal Fluminense (UFF)

Rua Passo da Pátria, 156 - 209, São Domingos, Niterói - RJ

cfsg1@bol.com.br

\section{RESUMO}

O presente trabalho tem por objetivo apresentar a plataforma computacional online SapevoWeb, implementação computacional do método multicritério ordinal Simple Aggregation of Preferences Expressed by Ordinal Vectors - Multi Decision Makers (SAPEVO-M). Este método pode ser caracterizado como uma nova versão do método tradicional SAPEVO (Gomes et al. 1997), porém abrangendo múltiplos decisores. O sistema SapevoWeb é um software livre desenvolvido em linguagem Python, de fácil operação e rápida resolução para problemas de decisão. Para ilustrar a utilização da plataforma é empregado um exemplo de ordenação de Corvetas para a Marinha do Brasil. Outra aplicação alternativa para o programa SapevoWeb é o cálculo do peso de critérios, para utilização combinada com outro método de apoio à decisão. O programa propõe a inovação da apresentação de valores absolutos associados à ordenação por ocasião da apresentação do resultado final, transmitindo a informação da distância relativa entra as alternativas ordenadas.

Palavra-chave: Sapevoweb; Método multidecisor SAPEVO-M; Software online livre; Apoio à Decisão Multicritério; ordenação de navio para Marinha do Brasil.

\section{ABSTRACT}

This paper presents the website SapevoWeb, computational implementation of the ordinal multicriteria method Simple Aggregation of Preferences Expressed by Ordinal Vectors - Multi Decision Makers (SAPEVO-M). This method is as a new version of the 
traditional SAPEVO method (Gomes et al. 1997), but covering multiple decision makers. The SapevoWeb system is free software developed in Python language, easy to operate and quick to solve decision problems. An example of ordering Corvettes for the Brazilian Navy is used to illustrate the use of the website. Another alternative application for the SapevoWeb program is the weighting of criteria for use in combination with another decision support method. The program proposes the innovation of the presentation of absolute values associated with the ordering at the presentation of the final result, transmitting the information of the relative distance between the ordered alternatives.

Keywords: Sapevoweb; Multi decision makers method SAPEVO-M; Free online software; Multiple Criteria Decision Analysis (MCDA); warship ranking for brazilian navy.

\section{Como Citar:}

TEIXEIRA, Luiz Frederico Horácio de Souza de Barros; SANTOS, Marcos dos; GOMES, Carlos Francisco Simões. Proposta e implementação em python do método Simple Aggregation of Preferences Expressed by Ordinal Vectors - Multi Decision Makers: uma ferramenta web simples e intuitiva para Apoio à Decisão Multicritério. In: SIMPÓSIO DE PESQUISA OPERACIONAL E LOGÍSTICA DA MARINHA, 19., 2019, Rio de Janeiro, RJ. Anais [...]. Rio de Janeiro: Centro de Análises de Sistemas Navais, 2019.

\section{INTRODUÇÃO}

Segundo Gomes e Gomes (2019), os métodos de apoio multicritério à decisão (AMD) são técnicas de assessoramento ao agente decisor (quer seja indivíduo, grupo de indivíduos, comitê de especialistas ou lideranças) para a tomada de decisões sobre problemas complexos, selecionado, avaliando ou ordenando alternativas mediante diferentes pontos de vista e dentro de critérios pré-estabelecidos.

O resultado pretendido em determinado problema de AMD pode ser identificado entre quatro tipos de problemática de referência, descritas por Gomes e Gomes (2019) como:

a) Problemática P. $\alpha$ - visa esclarecer a decisão pela escolha de um subconjunto de alternativas o mais restrito possível. Portanto, o resultado pretendido é uma escolha;

b) Problemática P. $\beta$ - visa esclarecer a decisão por uma triagem resultante da alocação de cada alternativa a uma classe (ou categoria). Portanto, o resultado pretendido é uma ação de classificação;

c) Problemática P.y - visa esclarecer a decisão por uma organização obtida pelo reagrupamento de parte ou de todas as ações em classes de equivalência, que são ordenadas de modo parcial ou completo, conforme as preferências do(s) decisor(es). Portanto, o resultado pretendido é um procedimento de ordenação ou ranking;

d) Problemática P. $\delta$ - visa esclarecer a decisão por uma descrição das ações e de suas consequências. Portanto, o resultado pretendido é um procedimento cognitivo ou uma descrição.

Para Gomes e Maia (2012), no entanto, estas problemáticas supracitadas não são completamente independentes umas das outras. Kuo (2017) destaca que, às vezes, a tomada de decisões torna-se uma tarefa difícil e complexa, em especial, por exemplo, quando se trata de encontrar a melhor alternativa, uma opção, uma escolha ou uma ação, classificando todas as alternativas na presença de múltiplos critérios, normalmente conflitantes.

Doumpos e Figueira (2018) e Yu et al.(2018) destacam que os métodos de ordenação podem ser classificados em duas categorias básicas: métodos cardinal ou ordinal. Gomes e Maia (2012) reforçam que a ordenação das alternativas (P.ү) pode servir de base 
para resolver um problema de escolha ou classificação. Segundo aqueles autores, muitos métodos multicritério, sobretudo aqueles desenvolvidos nos anos 70 e princípio dos anos 80, privilegiaram a problemática de ranking P.ү.

O método SAPEVO-M (Simple Aggregation of Preferences Expressed by Ordinal Vectors - Multi Decision Makers) representa uma nova versão do método de AMD ordinal SAPEVO proposto inicialmente por Gomes et al. (1997), para problemas especialmente do tipo P.ү. Esta evolução da versão original estendeu o emprego do método para múltiplos decisores.

Este artigo é composto de uma Introdução, seguido da descrição resumida do Método de AMD ordinal SAPEVO-M. Na terceira seção é explicado como ocorreu a implementação computacional da plataforma SapevoWeb e na quarta parte do artigo é exemplificada a utilização da mesma. As considerações finais dos autores estão registradas na Conclusão.

\section{MÉTODO ORDINAL SAPEVO-M}

Os métodos que empregam múltiplos critérios em auxílio à tomada de decisão têm um caráter científico e, concomitantemente, subjetivo, trazendo consigo a capacidade de combinar, de maneira ampla, as características consideradas importantes, inclusive as não quantitativas, com o objetivo de possibilitar a transparência e sistematização do processo referente aos problemas de decisão (Santos et al. 2017).

Segundo Gomes e Costa (2015), a ciência que engloba o AMD é caracterizada pela modelagem de problemas decisórios discretos à luz de pontos de vista diversos. Para aqueles autores, o AMD também se caracteriza por tratar de problemas que envolvam critérios qualitativos ou avaliações subjetivas.

O método SAPEVO-M, como nova versão do método ordinal SAPEVO (Gomes et al. 1997) original, possibilita a utilização de apenas um decisor. Além disso, introduziu um processo de normalização das matrizes de avaliação, incrementando a consistência do modelo. Evitando a necessidade de uma pré ordem dos critérios, e a mesma ser obrigatoriamente de consenso dos decisores.

Basicamente, o método consiste em dois processos. Preliminarmente, deve ser realizada a transformação da preferência ordinal entre critérios, a ser expressa por um vetor representando os pesos dos critérios. Em seguida, é feita a transformação ordinal da preferência entre alternativas dentro de um determinado conjunto de critérios, expressa por uma matriz. Uma série de comparações pareadas entre as opções quer seja critérios ou alternativas dentro de um determinado critério, denotam as informações de preferência individual de cada decisor.

Sejam ci e cj dois critérios dentro de um conjunto de critérios $C=\left\{c_{1}, c_{2}, \ldots c_{i}, \ldots, c_{j}\right.$ ,... \}, o grau de preferência entre eles é dado por $\delta c i c j$, no qual:

$\delta c_{i} c_{j}=1 \leftrightarrow c_{i} \cong c_{j}$, isto é, $c_{i}$ é tão importante quanto $c_{j}$;

$\delta \mathrm{c}_{\mathrm{i}} \mathrm{C}_{\mathrm{j}}>1 \leftrightarrow \mathrm{c}_{\mathrm{i}}>\mathrm{c}_{\mathrm{j}}$, isto é, $\mathrm{c}_{\mathrm{i}}$ é mais importante que $\mathrm{c}_{\mathrm{j}}$; e

$\delta \mathrm{c}_{\mathrm{i}} \mathrm{c}_{\mathrm{j}}<1 \leftrightarrow \mathrm{c}_{\mathrm{i}}<\mathrm{c}_{\mathrm{j}}$, isto é, $\mathrm{c}_{\mathrm{i}}$ é menos importante que $\mathrm{c}_{\mathrm{j}}$.

Uma escala de sete pontos expressa a relação entre as alternativas, na qual são mensuradas, relativamente, a importância entre cada opção. A partir da avaliação entre alternativas, é obtido uma matriz com a representação numérica correspondente. A relação entre a escala de preferência e o valor numérico é expressa na Tabela 1.

Seja D um conjunto de atores que decidem DM (Decision Makers), $D=\left\{\mathrm{DM}_{1}\right.$, $\left.\mathrm{DM}_{2}, \ldots, \mathrm{DM}_{\mathrm{k}}, \ldots, \mathrm{DM}_{\mathrm{n}}, \ldots\right\}$ que expressam suas opiniões sobre os critérios em ordem de preferência. Para um agente decisor $\mathrm{DM}_{\mathrm{k}}$, estas informações geram uma matriz de avaliação $\mathrm{MDM}_{\mathrm{k}}$. 
Tabela 1 - Relação entre a escala de preferência e valor numérico

\begin{tabular}{|c|c|}
\hline Preferência & Escala numérica \\
\hline$<<\ll 1$ & -3 \\
\hline$<\ll 1$ & -2 \\
\hline$\ll 1$ & -1 \\
\hline 1 & 0 \\
\hline$» 1$ & 1 \\
\hline$>» 1$ & 2 \\
\hline$>>» 1$ & 3 \\
\hline
\end{tabular}

A relação entre as duas escalas da tabela 1 permite a transformação da matriz $\mathrm{MDM}_{\mathrm{k}}=\left[\delta \mathrm{c}_{\mathrm{i}} \mathrm{c}_{\mathrm{j}}\right]$ em um vetor coluna $\left[\mathrm{V}_{\mathrm{i}}\right]$ de tal forma que:

$\mathrm{Vi}=\sum_{j=1}^{m}$, observando-se a escala 2 da tabela 1 para $\mathrm{i}=\{1, \ldots, \mathrm{m}\}$ e $\mathrm{k}=\{1, \ldots, \mathrm{n}\} . \mathrm{O}$ vetor $\mathrm{V}$ representa as preferências entre critérios de cada DM.

Este método prevê, também, terminado o processo de integração da matriz, a normalização do vetor resultante. Esta normalização garante a geração de valores não negativos, por meio da equação $v=\left[\left(a_{i j}-\right.\right.$ Mín $\left.a_{i j}\right) \div\left(\right.$ Máx $a_{i j}-$ Mín $\left.\left.a_{i j}\right)\right]$. Durante a normalização dos critérios, os menores valores, dos pesos dos critérios, assumem valor zero, sendo substituídos por um valor arbitrado de $1 \%$ do valor imediatamente superior; este procedimento deve-se ao fato de não fazer sentido que um determinado critério possua peso nulo. A escolha da fórmula de normalização foi feita após uma análise dos quatro métodos matemáticos de normalização.

De maneira análoga ao procedimento para obtenção do vetor peso dos critérios, dado um conjunto de alternativas, estas são comparadas par a par, à luz de cada critério. Desta forma, transforma-se a matriz $E=\left\{e_{i, k, l}\right]$ em um vetor coluna Ai, onde $e_{i, k, l}$ é o valor da comparação pareada da alternativa $\mathrm{a}_{\mathrm{k}}$ com a alternativa $\mathrm{a}_{1}$ dentro do critério I, para cada agente decisor DM. Logo, obtém-se o vetor coluna $\mathrm{A}$, onde $\mathrm{A}=\sum \mathrm{ADM}$ da matriz $\mathrm{M}$ (n X $\mathrm{m})$ ). Alterando-se o critério I na qual as alternativas foram avaliadas, procede-se novamente a comparação pareada e a integração do vetor, de modo que a matriz M seja toda preenchida.

Finalmente, o resultado da preferência entre as alternativas é expresso pelo vetor resultante da multiplicação matricial entre o vetor peso dos critérios $\mathrm{V}$ e a matriz de avaliação das alternativas $M$. As alternativas são, então, ordenadas em ordem decrescente dos valores numéricos obtidos, gerando-se o ranking desejado.

\section{SISTEMA COMPUTACIONAL SAPEVOWEB}

O sistema SapevoWeb foi desenvolvido a partir de uma parceria entre o corpo técnico do Centro de Análise de Sistemas Navais (CASNAV), um grupo de pesquisa do Programa de Pós-Graduação em Engenharia de Produção da Universidade Federal Fluminense (UFF) e um grupo de pesquisa do Programa de Pós-Graduação em Engenharia de Sistemas e Computação do Instituto Militar de Engenharia (IME).

A implementação computacional foi realizada em duas fases. Primeiramente, a codificação do algoritmo do método SAPEVO-M foi realizada. Na etapa seguinte, foi 
desenvolvido um programa web, para facilitar o acesso e utilização por pesquisadores, professores e estudantes. O resultado foi a disponibilização da plataforma denominada SapevoWeb, na qual a entrada de dados é feita a partir de um sítio da internet (www.sapevoweb.com).

Para Millman e Aivazes (2011), Python é uma linguagem adequada para ser utilizada em pesquisa acadêmica, embora não tenha sido desenvolvida, a priori, com este propósito. Fangohr (2015) destaca algumas qualidades de Python para este fim, como o foco na produtividade e legibilidade do código, possuindo suporte a pacotes hierárquicos, gerenciamento automático de memória e tipos de dados dinâmicos.

Dentre os diferentes padrões para desenvolvimento de páginas web, Python suporta o Hypertext Markup Language (HTML), que é uma linguagem de marcação utilizada para publicar páginas Web estáticas que são interpretadas nos navegadores. O código do SapevoWeb foi desenvolvido na linguagem Python, pelo uso do framework Django, e HTML. O programa permite a inclusão de um número inteiro suficientemente grande de decisores, critérios e alternativas, limitado apenas pela capacidade de processamento do servidor.

\section{EXEMPLO DE APLICAÇÃO: ORDENAÇÃO DE CORVETAS}

Para exemplificar a utilização do SapevoWeb, o mesmo será utilizado na ordenação de três navios de guerra de médio porte para a Marinha do Brasil (MB). Serão utilizados três modelos de corvetas, propostos por Santos et al. (2016), por ocasião de estudo para a seleção de um novo navio para a MB.

Desde o século XVIII, a Marinha opera na defesa das navegações no ambiente marítimo e fluvial. Além de desenvolver estratégias de inspeção e controle para a segurança do litoral brasileiro, a MB também realiza atividades cívico-sociais, com os Navios de Assistência Hospitalar que levam assistência médica e odontológica às comunidades carentes da Amazônia e Pantanal (Santos et al. 2018).

A Marinha do Brasil (MB) promulgou em 2019 um documento intitulado Política Naval (PN) cujo foco é, em conformidade com a END, estabelecer os Objetivos Navais, que devem ser alcançados pela MB, orientando o seu planejamento estratégico. Dentre outros pontos, a PN destaca a importância da Marinha em atuar em todos os oceanos, e no Atlântico Sul, principalmente, além de Vias Fluviais e Águas Interiores, garantindo os interesses nacionais (Marinha do Brasil, 2019).

Portanto, evidencia-se uma alta demanda por navios em plena condição de uso para suprir as necessidades da Marinha quando, como e onde for preciso. Em meio a esta ampla gama de tarefas, observa-se um natural desgaste dos meios navais e cresce a importância da renovação sazonal de sua frota.

Segundo Santos et al. (2016), a MB, alinhada à Estratégia Nacional de Defesa, almeja o fomento da construção naval militar nacional, em sinergia com o suprimento de suas necessidades. Dentro deste contexto, em que pesem as restrições orçamentárias e obstáculos políticos, a Marinha tem buscado desenvolver os seus navios de guerra com tecnologia própria.

A aquisição de um novo tipo de navio de guerra para uma marinha não deve ser tratada como um traço isolado, pois representa apenas um ponto dentro de um ciclo de conhecimento técnico e experiência operativa do poder naval daquele país. A escolha por um projeto inédito traz consigo os desafios inerentes ao ponto de partida de um novo ciclo. Desta forma, privilegia-se, sempre que possível, o contínuo aperfeiçoamento em modelos já existentes e que tenham sido amplamente utilizados e testados por aquela instituição.

Santos et al. (2016), propõem três opções viáveis de projetos de construção em 
território nacional de navios de médio porte para a MB. Primeiramente, um projeto de replicação de um modelo original de corveta nacional já em uso pela instituição (Corveta Classe Barroso - CCB); outra opção seria a construção de um novo modelo, contendo algumas pequenas modernizações em relação à CCB (Corveta de 2.600 toneladas Cv2600); ou, finalmente, o desenvolvimento e construção de outro modelo diferente com significativas atualizações (Corveta de 3.000 toneladas - Cv3000).

Tabela 2 - Comparação entre as características dos modelos de Corvetas

\begin{tabular}{|c|c|c|c|}
\hline \multicolumn{4}{|c|}{ Comparação } \\
\hline Características & $\begin{array}{c}\text { Barroso (*) } \\
\text { Configuração } 1\end{array}$ & $\begin{array}{c}\text { CV -2600 } \\
\text { Configuração } 2\end{array}$ & $\begin{array}{c}\text { CV-3000 } \\
\text { Configuração } 3\end{array}$ \\
\hline L over-all (m) & 103,4 & 115,00 & 118,00 \\
\hline L water-line $(\mathrm{m})$ & 96,30 & 105,00 & 108,00 \\
\hline B water-line $(\mathrm{m})$ & 11,40 & 13,00 & 13,50 \\
\hline$B \max (m)$ & Flare $7,5^{\circ}: 12,70$ & 15,00 & 15,50 \\
\hline$T(m)$ & 4,00 & 4,00 & 4,30 \\
\hline $\mathrm{D}(\mathrm{m})$ & 6,75 & 8,70 & 9,00 \\
\hline L/B (wl) & 8,45 & 8,08 & 8,00 \\
\hline LWl /D & 14,27 & 12,07 & 12,00 \\
\hline$T / D$ & 0,59 & 0,49 & 0,48 \\
\hline $\mathrm{Cb}$ & 0,53 & 0,48 & 0,48 \\
\hline Cp Long. & 0,67 & 0,622 & 0,622 \\
\hline Peso leve & 1710 & 1.815 & 2.030 \\
\hline Peso leve + Res.Proj. (ton) & 1.813 & 1.924 & 2.152 \\
\hline DWT (tons) & 418 & 589 & 728 \\
\hline Desloc. Máx. (tons) & 2.231 & 2.513 & 2.880 \\
\hline Desl.Máx. + SLA (tons) & 2.388 & 2.690 & 3.085 \\
\hline$B / D$ & 1,69 & 1,494 & 1,500 \\
\hline S plano d'água (m2) & 1.029 & 1.057 & 1.133 \\
\hline Seção mestra (m2) & 36,12 & 40,14 & 44,82 \\
\hline $\mathrm{GM}(\mathrm{m})$ & 1,70 & 1,68 & 1,68 \\
\hline Troll (seg) & 6,97 & 7,99 & 8,3 \\
\hline T pich (seg) & 5,19 & 5,40 & 5,48 \\
\hline S área molhada (m2) & 1.215 & 1.348 & 1.463 \\
\hline Propulsão (modo) & $(* *)$ Codog/Codad & Codad/Codoe & Codad/Codoe \\
\hline $\mathrm{PB} \max (\mathrm{MW})$ & Veloc 15Kts : 1,994 & 22,03 & 23,79 \\
\hline Raio de ação (n.m.)/15 & 4.000 & 9.330 & 10.660 \\
\hline Raio de ação (n.m.)/18 & $\mathrm{xxx}$ & 7.070 & 8.011 \\
\hline Fuel endurance (dias)/15 & 11 & 26 & 30 \\
\hline Fuel endurance (dias)/18 & $\mathrm{xxx}$ & 16 & 19 \\
\hline Autonomia (dias) & $(* * *) 30$ & 25 & 35 \\
\hline Tripulação (pessoas) & 150 & $100+20$ & $100+20$ \\
\hline Velocidade máx. (nós) & 27 & 28 & 28 \\
\hline SLR Vnax & 1,415 & 1,405 & 1,386 \\
\hline № de Froude & 0,452 & 0,449 & 0,442 \\
\hline Hélice ( dia/rmp/28kts) & $\mathrm{xxx}$ & 3,50 m/5 pás/285 RPM & 3,50 m/ 5 pás/ 292 RPM \\
\hline Geração Elétrica (KW) & 2.600 & $3.240 K W+408 \mathrm{KW}$ & $3.240 \mathrm{KW}+408 \mathrm{KW}$ \\
\hline Canhão principal & BA e $114 \mathrm{~mm} \mathrm{MK}$ & Oto Melara 76mm Sp & Oto Melara 76mm Sp \\
\hline Canhão secundário & Bofors $40 \mathrm{~mm}$ MK-3 & $2 \times$ Befors $40 \mathrm{~mm}$ MK-4 & 2x Befors 40mm MK-4 \\
\hline Mísseis ASuW & $8 \mathrm{x}$ Exocet SSM40 BI 3 & 8x Exocet SSM40 BI 3 & 8x Exocet SSM40 BI 3 \\
\hline Torpedos ASW & 2x III Raytheon MK-46 & 2x III Raytheon MK-46 & 2x III Raytheon MK-46 \\
\hline Helicóptero & $1 x \operatorname{lyn} x$ / AW 159 & $1 x \operatorname{lyn} x$ / AW 159 & 1x lynx / AW 159 \\
\hline Custo Inicial de Obtenção & US\$ 290 milhões & US\$ 310 milhões & US\$ 310 milhões \\
\hline Ciclo de vida 35 anos & US\$ 592 milhões & US\$ 633 milhões & US\$ 633 milhões \\
\hline
\end{tabular}




\begin{tabular}{|c|c|c|c|}
\hline $\begin{array}{c}\text { Tempo de Comissionamento } \\
\text { Primeira classe }\end{array}$ & 6 anos & $(* * * *) 8$ anos & $(* * * *) 8$ anos \\
\hline
\end{tabular}

Fonte: Adaptado de Vogt [2013]

A tabela 2 indica um comparativo entre as características dos três modelos. Cabe ressaltar que, no presente estudo, será desconsiderado o custo envolvido como critério para ordenação. Isto se deve, pois, devido à sua relevância, o mesmo será analisado como critério de veto. Deste modo, considera-se que os modelos a serem ordenados já passaram pelo filtro financeiro e são alternativas viáveis, dentro do limite orçamentário disponibilizado.

Com base no estudo do mapa mental proposto por Santos et al. (2016), ilustrado na Figura 1, e visando facilitar a ilustração gráfica e representação explicativa da utilização do SapevoWeb, os critérios foram compilados em quatro categorias: estrutura; máquinas; capacidade operacional; e poderio bélico.

Figura 1 - Mapa mental para estruturação do problema

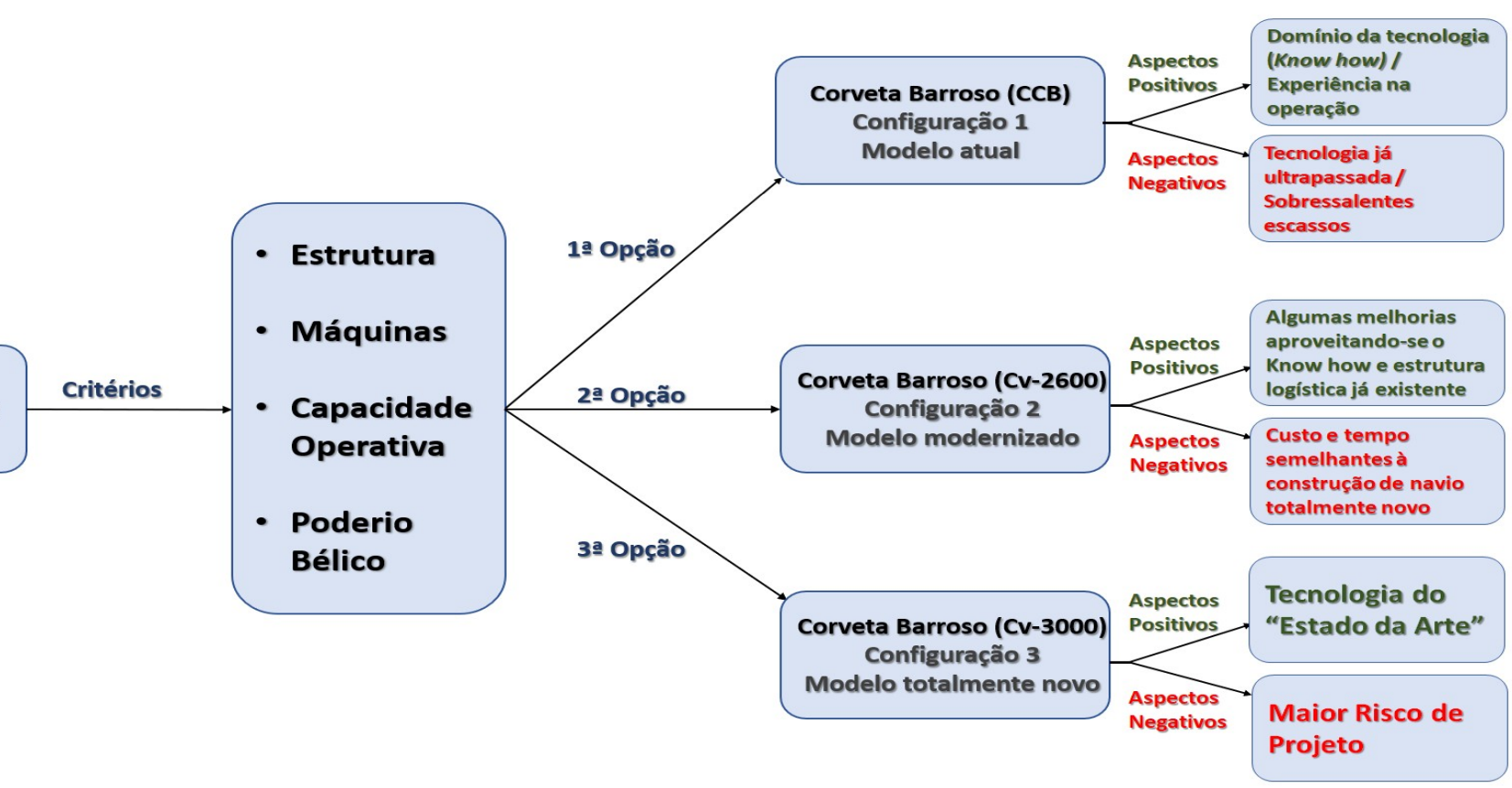

Fonte: Adaptado de Santos et al. (2016)

- Estrutura: combinação das características físico-estruturais da plataforma;

- Máquinas: combinação das características dos sistemas de máquinas principais e auxiliares;

- Capacidade operacional: autonomia, velocidade, raio de ação, transporte de tropa e endurance.

- Poderio bélico: combinação do poder combatente, incluindo armas, mísseis, sistemas de combate e de guerra eletrônica.

Para a ordenação entre os navios, será usado o método SAPEVO-M, a partir da plataforma SapevoWeb. A avaliação dos meios foi realizada, a convite dos autores, por três oficiais da Marinha do Brasil, ex-comandantes de navios e com larga experiência embarcados. 
Para utilização do SapevoWeb, faz-se necessária a inserção de dados por parte do usuário. Após acessar o endereço na internet, a primeira informação solicitada ao usuário é que o mesmo cadastre o projeto a ser iniciado, identificando-o por algum nome, conforme a Figura 2.

\section{SapevoWeb}

\section{Cadastrar Projeto}

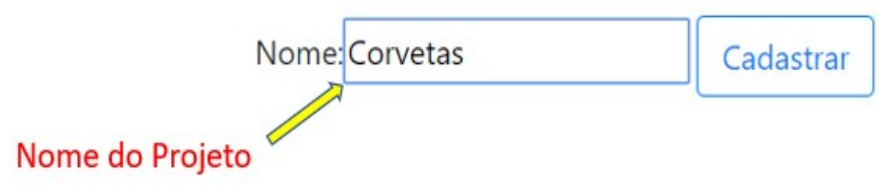

Fonte: www.sapevoweb.com

Após a digitação do nome, para confirmar a inserção de dados no sistema, deve-se pressionar a tecla "Cadastrar".

Como o SAPEVO-M é um método ordinal com múltiplos decisores, a tela inicial do projeto cadastrado solicita ao usuário a entrada dos nomes dos tomadores de decisão a serem considerados. A cada nome digitado, clica-se em "Cadastrar” para passar ao próximo nome, tantas vezes quantas forem o número de decisores, conforme mostrado na Figura 3.

Figura 3 - Tela para cadastrar Decisor 
SapevoWeb

SAPEVO-M. Simple Aggregation of Preferences Expressed by Ordinal Vectors - Multi Decision Makers

\section{Projeto Corvetas}

\section{Cadastrar Decisor}

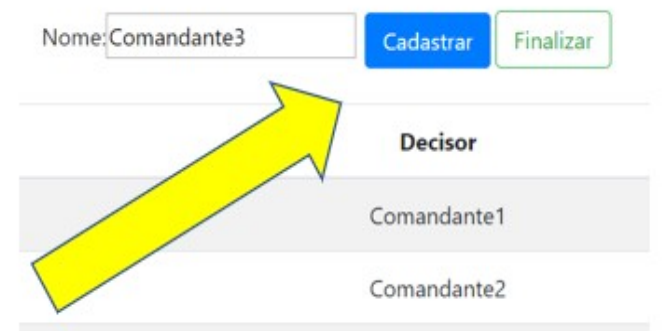

Fonte: www.sapevoweb.com

Os agentes decisores são cadastrados um a um, até que todos estejam inseridos no sistema. Então, para passar ao passo seguinte, clica-se no botão "Finalizar". No presente artigo, foram empregados três Decision Makers, a saber: "Comandante 1", "Comandante 2" e "Comandante 3", e após a inserção foi acionado o botão "Finalizar", conforme ilustrado na Figura 4.

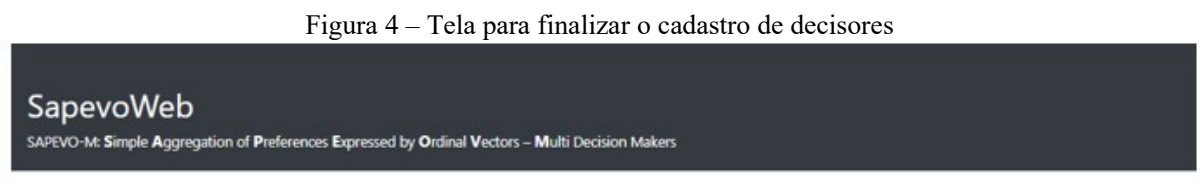

\section{Projeto Corvetas}

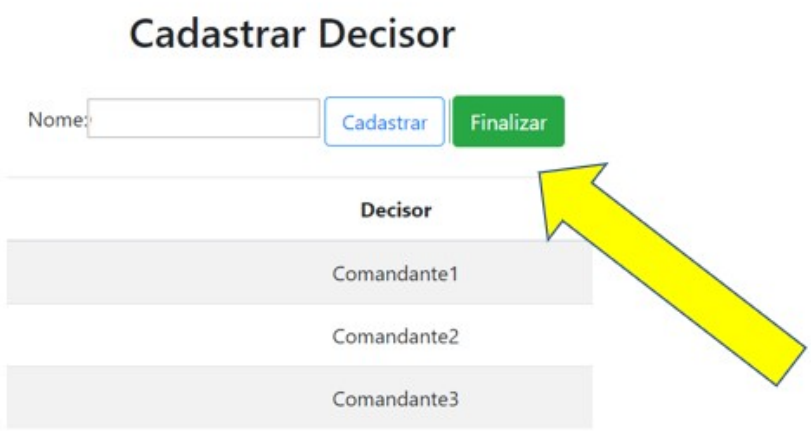

Fonte: www.sapevoweb.com

Posteriormente, analogamente, deverão ser inseridas manualmente pelo usuário as informações dos nomes dos critérios e das alternativas analisadas, conforme mostram as Figuras 5 e 6, respectivamente. 


\section{SapevoWeb Home}

\section{Projeto Corvetas}

\section{Cadastrar Alternativas}

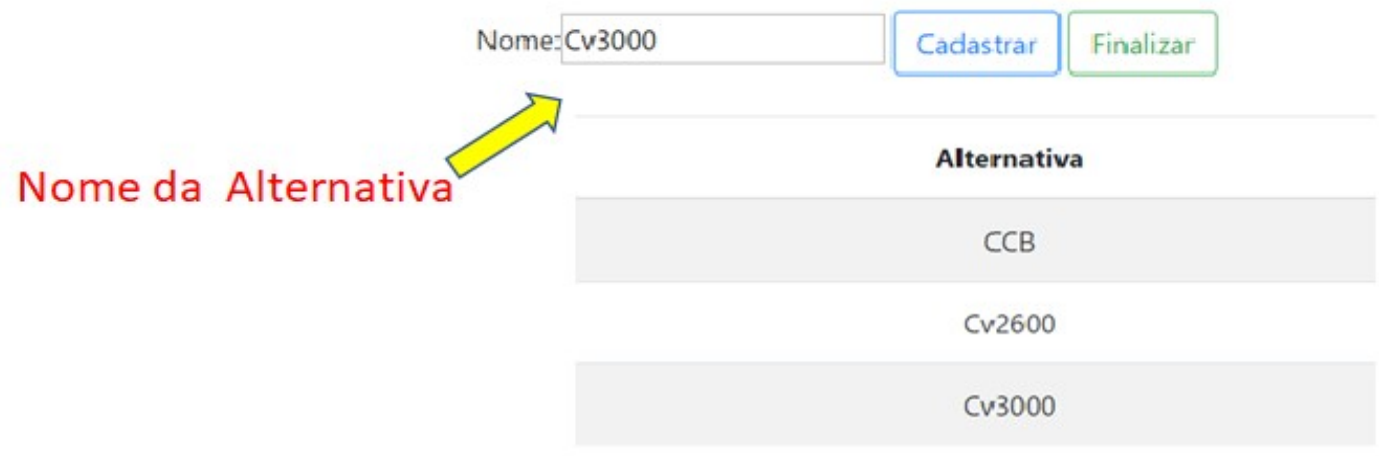

Fonte: www.sapevoweb.com

\section{SapevoWeb}

\section{Projeto Corvetas}

\section{Cadastrar Critério}

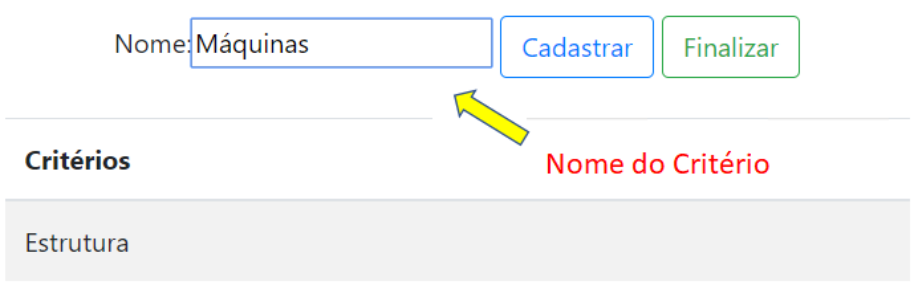

Fonte: www.sapevoweb.com

Caso algum dado tenha sido digitado incorretamente, por ocasião da inserção dos nomes dos decisores, alternativas ou critérios, sempre restará a opção de corrigir esta informação antes de finalizar. Clicando-se no próprio nome incorreto, abrirá uma nova janela para editar o nome e reescreve-lo da maneira correta. Nesta nova janela é possível "Salvar" ou “Cancelar” a edição, ao acionar os botões com as referidas ações. 
Para facilitar o entendimento, a Figura 7 ilustra que hipoteticamente tenha sido digitado o nome do critério "MáQQQuinas" ao invés de "Máquinas" e a Figura 8 apresenta a janela de edição que aparece ao se clicar no nome a ser corrigido. As Figuras 9 e 10 demonstram, respectivamente, a digitação correta do nome e o resultado final da edição.

Figura 7 - Tela com nome de critério digitado incorretamente

\section{SapevoWeb}

\section{Projeto Corvetas}

\section{Cadastrar Critério}

Nome: Cadastrar Finalizar

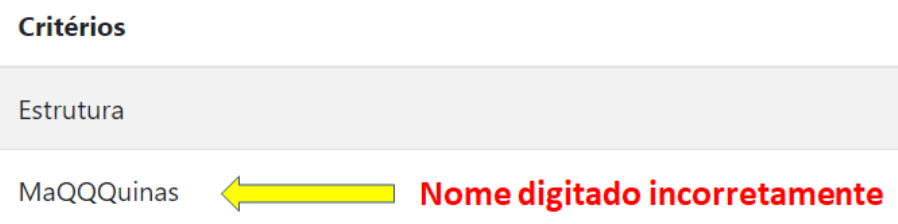

Fonte: www.sapevoweb.com

Figura 8 - Tela com janela de edição do nome do critério

\section{SapevoWeb}

\section{Projeto Corvetas}

\section{Cadastrar Critério}

Nome: Cadastrar Finalizar

\section{Critérios}

Estrutura

Fonte: www.sapevoweb.com 


\section{SapevoWeb}

\section{Projeto Corvetas}

\section{Cadastrar Critério}

Nome: Cadastrar Finalizar

\section{Critérios}

Estrutura

Máquinas

\section{Salvar Cancelar}

dirn

Fonte: www.sapevoweb.com

Figura 10 - Tela com o resultado da correção e inserção de outros critérios

\section{SapevoWeb}

SAPEVO-M: Simple Aggregation of Preferences Expressed by Ordinal Vectors - Multi Decision Makers

\section{Projeto Corvetas}

\section{Cadastrar Critério}

Nome: Poderio Bélicd $\quad$ Cadastrar Finalizar

\begin{tabular}{l} 
Critérios \\
Estrutura \\
\hline Máquinas \\
Capacidade Oper. \\
Fonte: www.sapevoweb.com
\end{tabular}

Após inserir todos os nomes de critérios, quando o usuário efetivar o clique no botão "Finalizar", o programa abrirá uma nova página HTML com os campos para avaliação, par a par, dos critérios, separados por cada decisor.

A avaliação é feita sobre um critério em relação a outro especificado, conforme 
ilustra a Figura 11.

Figura 11 - Tela com a escala de comparação pareada entre critérios

SapevoWeb

SAPEVO-M:Simple Aggregation of Prefereneres Eppressed by Ordinal Vectors - Mutiti Decision Makers

\title{
Projeto Corvetas
}

\author{
Avaliar Critérios \\ Qual é o decisor? Comandante1 \\ Estrutura VS Máquinas \\ Absolutamente Pior Muito Pior Pior Equivalente Melhor Muito melhor Absolutamente melhor
}

Fonte: www.sapevoweb.com

Deve-se atentar que a avaliação é feita a partir do critério mais à esquerda em relação ao outro mais à direita. Ou seja, no processo ilustrado acima na Figura 11, a avaliação realizada representou o grau de importância / relevância do critério "Estrutura” em relação ao critério "Máquinas'. A avaliação é feita através de uma comparação pareada, numa escala de sete estados, em que as expressões linguísticas equivalem aos graus de preferência apresentados na Tabela 1 . O sistema garante todas as combinações possíveis de comparação, sem repetição, conforme ilustrado na Figura 12.

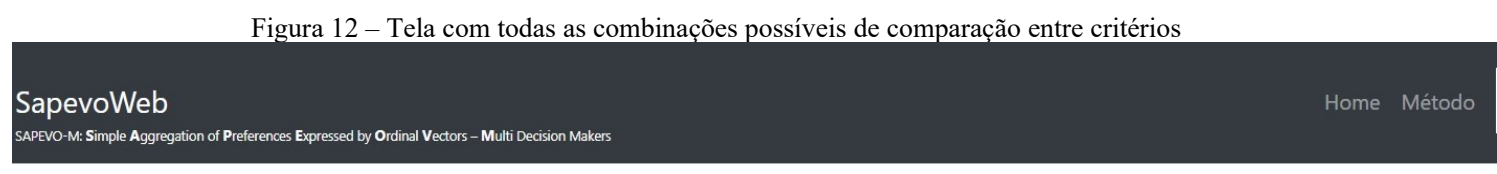

\section{Projeto Corvetas}

\begin{tabular}{|c|c|c|c|c|c|c|}
\hline \multicolumn{7}{|c|}{ Avaliar Critérios } \\
\hline \multicolumn{7}{|c|}{ Qual é o decisor? Comandante 1 } \\
\hline \multicolumn{7}{|c|}{ Estrutura VS Máquinas } \\
\hline Absolutamente Pior & Muito Pior & Pior & Equivalente & Melhor & Muito melhor & Absolutamente melhor \\
\hline \multicolumn{7}{|c|}{ Estrutura VS Capacidade Oper. } \\
\hline Absolutamente Pior & Muito Pior & Pior & Equivalente & Melhor & Muito melhor & Absolutamente melhor \\
\hline \multicolumn{7}{|c|}{ Estrutura VS Poderio Bélico } \\
\hline Absolutamente Pior & Muito Pior & Pior & Equivalente & Melhor & Muito melhor & Absolutamente melhor \\
\hline \multicolumn{7}{|c|}{ Máquinas VS Capacidade Oper. } \\
\hline Absolutamente Pior & Muito Pior & Pior & Equivalente & Melhor & Muito melhor & Absolutamente melhor \\
\hline \multicolumn{7}{|c|}{ Máquinas VS Poderio Bélico } \\
\hline Absolutamente Pior & Muito Pior & Pior & Equivalente & Melhor & Muito melhor & Absolutamente melhor \\
\hline \multicolumn{7}{|c|}{ Capacidade Oper. VS Poderio Bélico } \\
\hline Absolutamente Pior & Muito Pior & Pior & Equivalente & Melhor & Muito melhor & Absolutamente melhor \\
\hline
\end{tabular}


Depois de inserida a avaliação do $1^{\circ}$ agente decisor, deve-se clicar no botão “Avaliar” quando o sistema irá gerar uma nova janela para que seja inserida a avaliação do decisor subsequente. Ao término das avaliações dos critérios, analogamente, deve ser feita a avaliação par a par das alternativas, por cada decisor à luz de cada critério. A Figura 13 visa explicar o layout da página em que foram inseridas as impressões do avaliador "Comandante 2", sobre o desempenho / importância da alternativa "CCB” em relação à alternativa “Cv2600”, dentro do previsto para o critério "Estrutura”.

Figura 13 - Tela com a comparação de alternativa dentro de critério

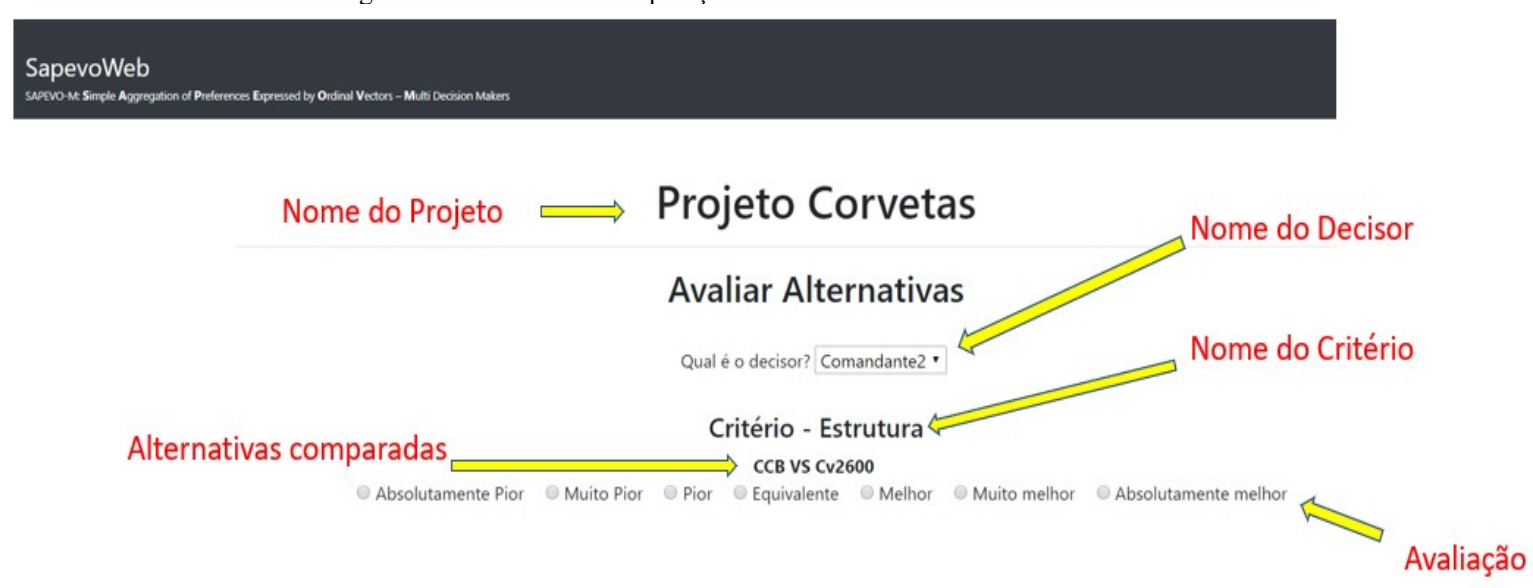

Fonte: www.sapevoweb.com

Após inseridos os dados de avaliação de todos os agentes decisores em todos os critérios, ao se clicar em "Avaliar” o resultado é apresentado. Para o exemplo em questão neste trabalho, a Figura 14 demonstra o peso dos critérios e a ordenação dos navios.

Figura 14 - Tela com resultado final do peso dos critérios e ordenação das alternativas 


\title{
Resultado | Projeto Corvetas
}

\section{Pesos}

\author{
Critério - Estrutura - 0.013333333333333332 \\ Critério - Máquinas - 1.0033333333333334 \\ Critério - Capacidade Operativa - 1.3333333333333333 \\ Critério - Poderio Bélico - 2.0

\section{Ordenação} \\ $1^{\circ}--$ Cv2600 -- 6.3566666666666665 \\ $2^{\circ}--$ Cv3000 -- 5.125238095238096 \\ $3^{\circ}--$ CCB -- 1.750219 \\ Fonte: www.sapevoweb.com
}

Corroborando com o que havia sido proposto por Santos et al. (2016), através do método AHP, os três especialistas, utilizando o sistema SapevoWeb baseado no método ordinal SAPEVO-M, concluíram que a opção Cv2600 seria a melhor ordenada em relação às demais.

\section{CONCLUSÃO}

Com o objetivo de que os resultados de problemas complexos possam ser facilmente obtidos e os pesquisadores possam focar em outras etapas do processo, o sistema SapevoWeb surge como uma importante ferramenta. A disponibilização online desta plataforma de auxílio à decisão multicritério possibilita a difusão de um novo método ordinal para a comunidade acadêmica.

Cabe ressaltar que a interface gráfica amigável e resultados em tempo computacional reduzido garantem a facilidade de utilização do programa SapevoWeb. Ademais, obtêm-se com simplicidade e rapidez os valores numéricos para os pesos dos critérios, ampliando a utilização desta plataforma apenas até a fase de obtenção dos pesos, associada a outro método de apoio à decisão.

Finalmente, uma novidade introduzida pela plataforma SapevoWeb é apresentar o resultado final da ordenação expressando valores numéricos associados à ordem de cada alternativa. Ou seja, embora o método SAPEVO-M seja um método ordinal multicritério para múltiplos decisores, a informação transmitida ao decisor não se restringe à disposição das alternativas em um ranking. Indo além, o software SapevoWeb permite conhecer o quanto uma alternativa ficou melhor ordenada, relativamente, do que outra, passando uma informação adicional ao tomador de decisão. 


\section{REFERÊNCIAS BIBLIOGRÁFICAS}

[1] Doumpos, M.; Figueira, J. R. A multicriteria outranking approach for modeling corporate credit ratings: an application of the Electre Tri-nC method. Omega (in press):1-15. (2018)

[2] Fangohr, H. Introduction to Python for Computacional Science and Engineering. Faculty of Engineering and the Environment, University of Southampton. (2015). Disponível em:

$<$ https://www.southampton.ac.uk/ fangohr/training/python/pdfs/Python-forComputational-Science-and-Engineering.pdf> Acesso em: 20/04/2019.

[3] Gomes, C. F. S. e Costa, H. G. Aplicação de métodos multicritério ao problema de escolha de modelos de pagamento eletrônico por cartão de crédito. Produção (São Paulo. Impresso), v. 25, p. 54-68. (2015).

[4] Gomes, C.F.S. e Maia, A. C. C. Biomassa como alternativa para o fornecimento de energia. Pesquisa Operacional para o Desenvolvimento, v.4, n.1. (2012).

[5] Gomes, L. F. A. M., e Gomes, C. F. S. Princípios e métodos para a tomada de decisão: Enfoque multicritério (6a ed.). São Paulo: Atlas. (2019).

[6] Gomes, L. F. A. M., Mury, A. R., Gomes, C. F. S. Multicriteria ranking with ordinal data Systems Analysis Modelling Simulation. (1997).

[7] Kuo, T. A modified TOPSIS with a different ranking index. European Journal of Operational Research, v.260, p.152-160. (2017).

[8] Marinha do Brasil. Política Naval. (2019) Disponível em < https://www.marinha.mil.br/politicanaval > Acesso em: 20/04/2019..

[9] Millman, K. J. e Aivazis, M. Python for Scientists and Engineers. Computing in Science \& Engineering, 13(2). (2011). Doi:10.1109/MCSE.2011.36

[10] Santos, M. Gomes, C. F. S. Oliveira, A. S. Costa, H. G. Uma abordagem multicritério para seleção de um navio de guerra de médio porte a ser construído no Brasil. In: XLVIII Simpósio Brasileiro de Pesquisa Operacional, 2016, Vitória, Espírito Santo. p.507 a 518. (2016)

[11] Santos, M. Pimenta, V. A. Lauria, R. L. Carvalho, F. B. Souza, J. A. Uma estratégia de otimização em navios de guerra da Marinha do Brasil. Brazilian Journal of Development, v. 4, p. 3015-3034. (2018)

[12] Santos, M., Rodriguez, T. O., Quintal, R. S., Dias, F. C. e Reis, M. F. Emprego de Métodos Multicritério para apoio à decisão em empreendimento turísticos: o caso do Hostel Ocean inn Rio. CULTUR: Revista de Cultura e Turismo, v. 11, p. 87-107. (2017).

[13] Teixeira, L. F. H. S. B., Santos, M. e Gomes, C. F. S. SapevoWeb Software (v.1). (2018). Disponível em: <http://www.sapevoweb.com> Acesso em: 30/04/ 2019.

[14] Yu, X., Zhang, S., Liao, X. e Qi, X. ELECTRE methods in prioritized MCDM environment. Information Sciences, v.424, p.301-316. (2018) 
[15] Vogt, R. Corvetas Sucessoras Da Barroso: Comparação De Dois Tipos De Obtenção. Revista Marítima Brasileira/ Serviço De Documentação Geral Da Marinha. v.133, N.04/06. Abr./Jun - Rio De Janeiro: Marinha Do Brasil. (2013) 